\title{
WMLP: Web-based Multi-Layer protocols for Emergency Data Transmission in Mobile Ad Hoc Network
}

\author{
K. Saravanan ${ }^{1}$, S. Anthoniraj ${ }^{2}$, S. Kumarganesh ${ }^{3}$, T.Senthil Kumar ${ }^{4}$, K. Martin Sagayam ${ }^{5}$ \\ ${ }^{1}$ saravklvn@gmail.com, ${ }^{2}$ anthonirajcse@gmail.com, ${ }^{3}$ saikgss@gmail.com, drtsenthilkumar2018@gmail.com, \\ martinsagayam.k@gmail.com \\ ${ }^{1}$ Professor, Department of Information Science and Engineering, New Horizon College of Engineering, \\ Bangalore, India. \\ ${ }^{2}$ Associate Professor, Department of Computer Science and Engineering, MVJ College of Engineering, \\ Bangalore, India. \\ ${ }^{3 \&}$ Department of Electronics \& Communication Engineering, Siddharth Institute of Engineering and \\ Technology, Puttur, Andhra Pradesh, India \\ ${ }^{5}$ Department of Electronics \& Communication Engineering, Karunya Institute of Technology and \\ ABSTRACT \\ Sciences, Coimbatore, India
}

Mobile Ad Hoc Network (MANET) is an Emergency transportable Ad-Hoc network, which is a significant module of the Internet of Effects and has been extensively practiced in smart transportation systems in new existences. In this proposed system using Web-based Multi-Layer protocols for Emergency Data Transmission (WMLP), Dynamic multi-priority message order management method it is used to reduce the data transmission delay in entire networks. To fulfill the real-time limitations of emergency data, we proposed WMLP, a novel Networks web-like transmission mechanism for emergency data. We create a spiderweb-like perfect to limit the exact area. Source nodes send out demand terminus nodes send confirmed host in this controlled area to develop well-organized broadcast paths. The dynamic multi-priority file administration method it is managed some of packets level by level. In this protocols mining, necessary packs and giving the first importance emergency messages are function and considered for transmission failed packages also. When transmitting packets, we are using restricted containers strategy to choose the next hop.

Keywords :

Mobile Ad Hoc Network, Web-based Multi-Layer protocols, Emergency Data Transmission, Dynamic multipriority message queue.

\section{INTRODUCTION}

Mobile ad hoc networks recently since they have the great military and commercial potential. Portable specially appointed systems were unmistakable by a method for the gathering of remote frameworks that apply multi-jump radio imparting and are proficient of working without the help of any settled foundation (subsequently, they are likewise rung set fewer frameworks). The term $\backslash$ impromptu" proposes that it is a system perceived for a specific, 
regularly spontaneous administration adjusted to demands. Thus, the single remote impromptu framework is set up for a limited collectible, and the conventions tuned to the demand. The request might be versatile, and the circumstance may change vigorously. On the off chance that hubs are moveable, the framework is called as Mobile Ad-hoc Networks. In this way, the specially appointed conventions need self-organize to control the setting, movement, and mission changes. Spurred by theincreasing concentration in ad hoc networking, many regular activities, and commercial standards. Within the MANET working group was born and required to regulate courseplotting protocols for wireless ad hoc networks. The subcommittee dependable a medium access protocol that was two founded on error evading. HIPERLAN and Bluetooth areother values that addressed and advanced ad hoc networking[16][17].

\section{A. Applications of Mobile Ad Hoc Networks}

Wireless ad hoc networks, owing to their rapid and carefully less difficult placement, possess goals that are very dissimilar from moveable communication and Internet get to bastion. They find requests in several areas. The first category of appeals is to set up foundations for specific, adjusted, extemporary demands in parts where there is no prior set-up, e.g., battleground, rainforest pieces of training, or the forestry or lumber manufacturing. The second gathering of conceivable necessities is to set up transportations where the help has fizzled, e.g., seismic tremor saves. The third sort of requesting is invigorated by the absence of reasonable, minimal effort substructure, e.g., sensors disseminated throughout a city for nuclear detection, a support less network of notebook processors in a consultation or campus setting[19][20][21].

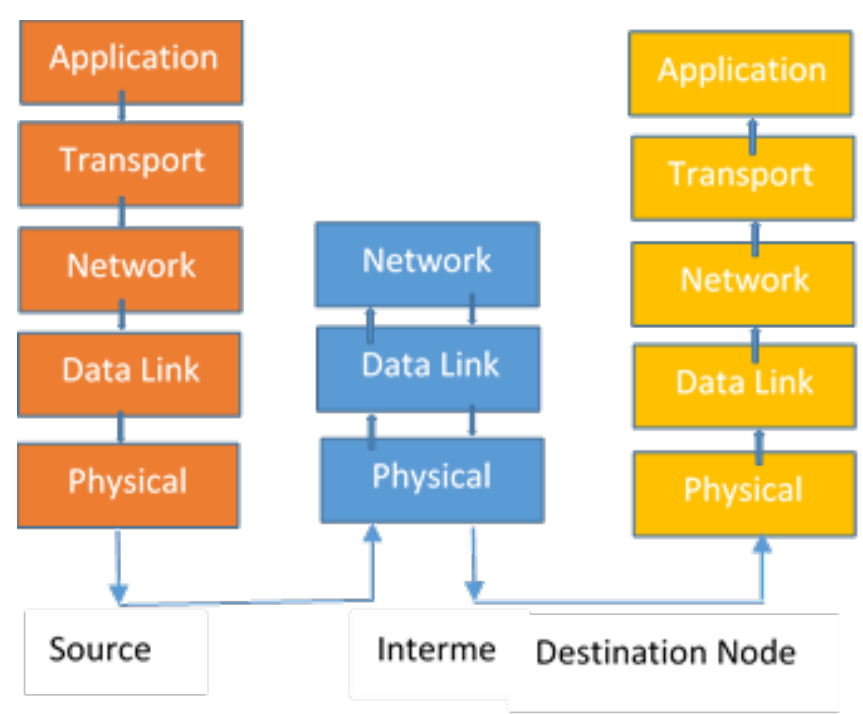

Figure 1Data transmission process in mobile networks

From figure1 the physical layer determines to efficiently transmit and receive data bits with as few faults as possible. It handles inflection, error coding, transmission, and reception. Because energy conservation is critical in MANET, we will focus on power control at the physical layer. The data link layer group's data bits into frames, 
and handles frame errors and controls the flow of frames. It is needed to coordinate the transfer of information 6 in any of the several ways, such as one-to-one (unicast), one-to-many (multicast), one-to-all (broadcast), or manyto-one (multi-access) within the radio range as determined by the physical layer. It is the accountability of the data link layer to perform error correction for irregularities happening in the physical layer. The data link layer usually defined as having two layers first one is logical link control and the second one is the medium access control layer [18].

\section{B. Selection of Best Path}

In this mobile network communication nodes between the distance of the conceivable straightway between all hubs in the system whenever and marks all packs with this way separate when they are made. In this system the change between this straight way length and the length of the approaches taken by information bundles. A distinction of 0 implies the ampule took the briefest form, and a change more prominent than 0 assigns the number of new jumps the bundle ordinary. Both DSDV-SQ and DSR utilize courses near best. TORA and AODV-LL each have the first tail, taking up to at least four bounces longer than best for a few parcels, even though TORA was not expected to discover straight ways. For space reasons, adds up to the information from all respite times into one diagram. At the point when the data is divided out by stop time, DSDV-SQ and DSR do despite delay time, with no measurable critical change in the optimality of steering regarding hub suppleness rate. TORA and AODV, then again, each demonstrate a first adjustment concerning stop time out there of the courses they utilize concerning the briefest possible ways. At the point when hub portability is low, they use maps that are seriously nearer to the possible straight directions than when swells are moving [22][23].

\section{Features of Mobile Ad Hoc Networks}

A wireless ad hoc network is a group of possibly moveable nodes that wish to connect, but have no secure substructure available, and have no pre-determined civilization of available links. Individual nodes are accountable for animatedly learning which other nodes they can right connect. A critical supposition is that not all nodes can directly connect with each other, so they are obligatory to relay packs on behalf of other nodes to deliver data crossways the network. A significant feature of wireless ad hoc networks is that rapid changes in connectivity and link Topographies presented due to node flexibility and power control applies. In the following, we appraisal main physiognomies of wireless ad hoc networks in part [24][25].

\section{Mobility}

The portability of a node is not a required condition for impromptu remote systems. For instance, the nodes conveyed for periodic checking of information determination in versatile systems properties are not required to be convenient. Some of the mobile nodes rapidly repositioned and moved on networks. The fast conveyance in territories with no infra-structure frequently proposes that the clients must investigate a city and possibly shape groups and oversee among themselves to make the team. We can have individually enhanced portability, gather versatility, motion along preplanned courses, and so on. The portability model can affect the determination of a 
steering course of action and would thus be able to impact introduction [17][26].

\section{E. Scalability}

Wireless ad hoc networks in scalability can generally be defined as whether the system can provide an appropriate level of service to packets uniform within sight of countless in the order. In some requirements, for example, sizeable recyclable sensor constituents, battlefield placement, the remote specially appointed system can develop to a few thousand nodes. For remote \substructure" systems cell organize, versatility is taken care of only by a positioned development. The restricted adaptability of framework systems can likewise be effectively moderate utilizing versatile IP or neighborhood handoff strategies. Inconsequentiality, feeling of the extraextraordinary event and the absence of secure introduction, unadulterated remote specially appointed systems do not demeanor portable IP or a sheltered progression structure. In this way, advance, together with a broad scale is a standout amongst the most hazardous challenges in remote specially appointed frameworks outline [18][27].

\section{F. Multi-Hopping}

A multi-hop network is a network data communication using multipath using to a source to the several nodes receivers' nodes. Wireless ad hoc networks involve self-directed nodes that cooperate to transport data. Generally, these nodes performance as end systems and routers at the same time. Because of most mobile ad hoc networks' mobility and scalability nature, they frequently display many bounces for hindrance mediation, extend reuse, and vitality safeguarding [19][[28].

\section{G. Medium Access Control}

The most for the most part Carrier Sense Multiple Access (CSMA) Macintosh plan and its varieties For example, CSMA with parcel misfortune and crashes find (CSMA/Album) created for wired systems techniques can't be explicitly utilized in the remote system in light of the fact that the remote correspondence channel is vitally inclined to blunders, the topology is for the most part arbitrary, and remote impromptu systems have their exciting issues, for example, the concealed terminal issue and the uncovered terminal issue, Shrouded terminal problem The covered terminal issue is called from in remote specially appointed systems. This issue happens when parcels creating from two or other sender nodes, which are not classified the straight transmission scope of each other, crash at a typical earpiece hub. It requires retransmission of packets. Hence, the presence of hidden positions can meaningfully reduce the throughput of a MAC protocol used in wireless ad hoc networks. Exposed-terminal problem Exposed workstations, the nodes that are in the broadcast range of the sender of an on-going session, are rejected from making a transmission. To improve the productivity of the MAC protocol, the exposed nodes should be allowed to communicate in a controlled fashion without creating a collision to the on-going data transfer. In wireless networks, the MAC protocol must struggle for access to the channel while at the same time evasion possible collisions with head-to-head nodes. How to resolve confects among different nodes for channel access and to ensure fair and efficient resource sharing at the same time makes the MAC protocol design a stimulating task for wireless ad hoc networks [20][29]. 


\section{LITERATURE REVIEW}

Contained Least Crossing Tree-Based Multicast Steering with Vitality Proficient Unmistakable Conveyance in Specially appointed and Sensor Systems We current a constrained geographic multicast to conspire, STEAM, considering the working of a nearby least spreading over trees (MSTs) that requires information just on single-jump neighbors. A message duplication influenced when the MST correspondence the present hub and the arrangement of goals has a portion of the points of interest began at the current center [1][23]. Ongoing Rate and Course Adjustment with Productive Connection Quality Evaluation for Multi-Bounce Systems, correct, and speedy bundle conveyance proportion (PDR) appraisal, utilized as a part of surveying remote connection brilliance, is a precondition to enhancing the execution of multi-jump and multi-rate remote systems. Lamentably, contemporary PDR estimation strategies, i.e., reference point-based bundle tallying in Evaluated Transmission Time and Expected Transmission Tally measurements, have inadmissible execution. We characterize all possible connection quality estimation (LQE) sources and partner them diagnostically against our plan, demonstrating the predominance of our approach. In this way, a novel PDR estimation technique considering SNR profiles and particular transmission bundles [2][17][19].

A powerful SNR evaluates for remote sensor arrange devouring Kalman channel, in numerous WSN entries, the accessibility of humble yet exact estimation of the RF position esteem is indispensable. In any case, due to evaluate clamor and blurring impacts, it is commonly surveyed through test or instruction-based techniques, which result in high dynamism ingesting or high overheads. We laud to influence utilization of data to end among pointers if by the framework to advance the gauge of the connection perfection. A channel-based arrangement is utilized because of its bent to give an exact appraisal of the un-indicated zones of quick clamor comments [3]. A Vitality Cognizant Directing in Remote Impromptu Systems, Online vitality mindful steering in remote frameworks is the blunder discovering vitality proficient courses that system lifetime's boost encounter because of future message streams. To enhance organize lifetime, the ways for message stream chose such that the aggregate vitality consumed along the course limited while staying away from control exhausted nodes. Find ways which expend the least power and discovering ways which do not utilize drained vitality nodes prompt clashing articles [4][18].

Least Vitality Trustworthy Ways Utilizing Undependable Remote Connections. In the interim, propels in real strategies are consistently expanding different remote correspondence stations to a detonating client populace. Proficiently taking care of disappointments in primitive conditions, accordingly, accept focal significance. In numerous situations, the outline of distant correspondence conventions guided by two necessities. Vitality effectiveness and versatility to parcel misfortunes. Indeed, even under ideal conditions, different elements, such as blurring, interfering, multi-way impacts, and crashes, prompt significant misfortune rates on remote connections [5][19]. Dynamic Electric Vehicle Reenactments, Propagation of systems nodes, half breed vitality nodes, and design in crossbreed vitality nodes of transportation over driving developers inside a full, powerful crossover and vitality nodes test system requires vitality models fit for foreseeing condition of-charge, faces, and active conduct of various battery writes. A vitality shows skilled at replicating flawless materials, nickel-metal hydride. A systems administration testing topology was intended to quantify the anticipated parameters of the vitality display for all multipath steering writes and great counterfeit records with a modified source and load creation [6][18]. 
Putting the best number of sinks in sensor systems for arranging expansion lifetime, Because of the effort of together deciding the ideal measure of a skillet and formulating a steering convention to expand the system lifetime, in this segment, we examined a heuristic for it. We deteriorate the issue into two sub-issues: finding the ideal number of sinks and their areas to such an extent that every sensor can achieve a dish without any than number jumps and be building a heap adjusted backwoods to amplify the system lifetime [7][19]. Versatile course steering close to a moveable sink for enhancing life in sensor systems we characterize organize lifetime as the period for the primary hub to come up short on its vitality saved. While surveying this amount, we change over the issue of augmenting system lifetime to a min-max problem concerning the radio vitality ingesting of partitioned nodes [8][18].

Dispersed centered lifetime directing in WSN because of regularization, a significant portion of the gadget nodes are vitality nodes. The constrained measure of vitality in every hub is one of the squares. A direct technique is to limit the power expended to convey a parcel to the end. To overpower this issue, various vitality mindful or vitality constrained Calculations. The run of the mill clarification is to utilize the straightway with interface costs equivalent to the power necessary in each connects to diffuse a parcel [9]. A deride demonstrate for the lifetime of remote sensor systems; remote sensors have gotten increased regard in the before years because of their reverence and cost-viability when they are utilized as a part of a brutal environment. They have been used as a part of numerous solicitations including soldierly entries, native applications, home applications, and wellbeing demands. Continuously are many costs dynamic and immediately sorted out in the unforgiving environment, they are defective by the power realistic completed their life cycle [10][19].

Various leveled conspire based intrusion location framework for a remote specially appointed sensor arrange, in this, is accomplished in some first looks in to. Be that as it may, the security work utilized for impromptu remote systems cannot be sorted out straightforwardly for WSNS since of their hierarchical imbalance. Initial, a specially appointed system, each hub is generally held and come to by a human client. While in a gadget arrange, every one of the nodes is self-representing, and a message is apt by the base station. Second, registering assets and arrangement are more unnatural in sensor nodes than in specially appointed nodes [11][20]. Vitality skill in specially selected systems, utilizing the top calculation is the lowermost id conventions which are bunching calculation. It depicts which nodes will execute as group heads and directs the nodes that establishment the bunch. Cover characterizes the nodes which will go about as bunch heads. What is more, played to cover the nodes. We appoint a single id to every hub in the system. The cover methodology chooses the hub self-assertively with the bottommost id as the bunch head and declares every one of the nodes private the scope of this group head as its individuals [12].Programmed informing frameworks, a full state technique is the place nodes know about their topology, its state, propelling choices. It needs the server to record the arrangement so they can be reviewed or continued. Two fundamental classes are interface state and separation - vector. A case of connection state is OLSR an example of the separation vector is DSDV. Both these conventions are proactive, which approaches to every single close-by hub in the framework and limits the underlying deferral [13]. Sunrise: vitality proficient information gathering in WSN with portable sinks, with the fairminded to amplify the lifetime of a static sensor organizes, we center on outlining dynamic directing plans for the static sensor arrange by beautiful, versatile sinks into account. We broadly look at and evaluate the vitality utilization and defer execution for information aggregation and accumulation with separate moveable sinks. Our conventions 
are novel in honest models with limited adaptability, which is the outcome from various topographic surroundings in large-scale demands. We likewise utilized a few qualified steering plans for information blend, to limit the conveyance delay while mishandling the system age [14]. Examination of Various Security Assaults in MANETS on Convention Stack an Audit, the principle of a derisive hub is to upset the ordinary procedure of the directing convention. The effect of such assault is expanded when the report happens between neighboring nodes. Foreswearing of Administration (DoS): These kinds of dangers delivered a malignant activity with the assistance of surrendered nodes that structures extreme security dangers. Within sight of bartered nodes, it is trying to recognize the arranged steering [15].

\section{IMPLEMENTATIONS OF PROPOSED SYSTEM}

Mobile Ad Hoc Network (MANET) is an Emergency transportable Ad-Hoc network, which is a significant module of the web link and has been extensively practiced in brainy transportation schemes in new existences. For large scale MANETs, it is substantial to design efficient transmission arrangements for time-critical emergency data. Greedy Perimeter Coordinator Routing Protocol is typical for map-based, but they do not consider the QoS of the broadcast web link and hence are not suitable for emergency data transmissions Neural networks protocols and situation-aware protocols are more appropriate for emergency positions. In this case, Web-based Multi-Layer protocols (WMLP) used to Emergency Data Transmission in MANETs in using additional search and savior and military data achievement procedures in rough terrain, among others. Then additional one method is Dynamic multi-priority message queue organization method it is used to condense the data transmission delay in whole networks. WMLP is a feed-forward false neural net that produces a set of productions from a set of inputs. MLP in the wireless system is based on the data models, constructed of the different types of input vector $\mathrm{x}$ and the M-models desired output vector $\mathrm{d}$, called terminus.

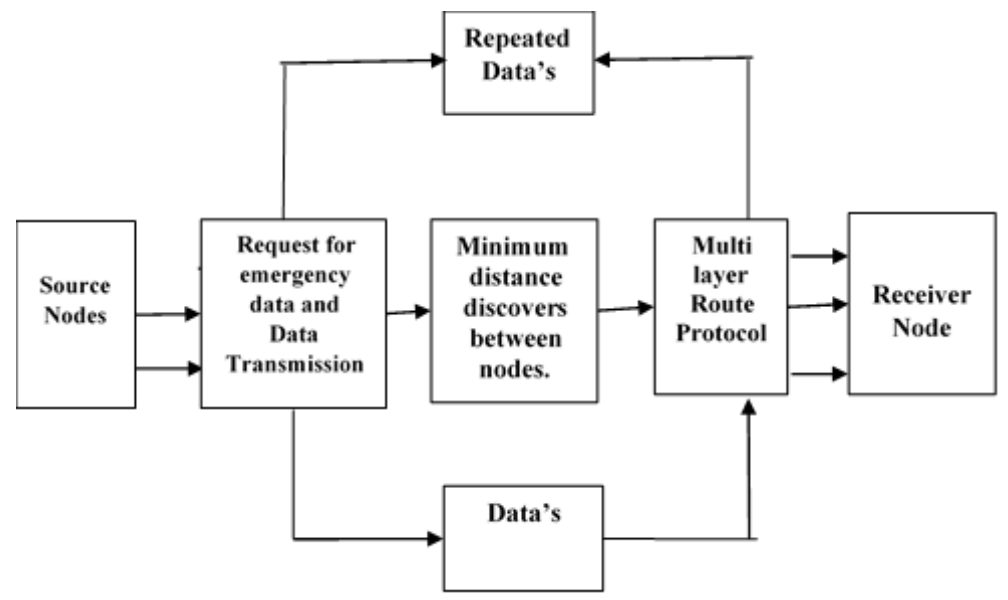

Figure 2. Sorting of root path in ad hoc networks

A routing protocol builds for use in mobile ad hoc networks. The contract divides the nodes of the ad hoc network into some overlapping or disjoint two-hop-diameter network division using a distributed method. The cluster-based 
planning was devised to minimize the inundating of route discovery packets. This kind of building is most suitable for large networks with several nodes. The complete system is alienated into many touching or disjoint two-hopdiameter root path. A cluster head is designated for each cluster to uphold cluster membership information. A bunch is recognized by its $\mathrm{CH}$ address. Inter-cluster itineraries are exposed dynamically using the cluster association material kept by each $\mathrm{CH}$. By meeting nodes into sets, the protocol ingeniously reduces the flooding circulation during route detection and speeds up this process. A node compliments itself as being in a cluster if it has a bidirectional link to the head of the group. In the present method of CBRP, a minimized node is automatically informed to the central station.

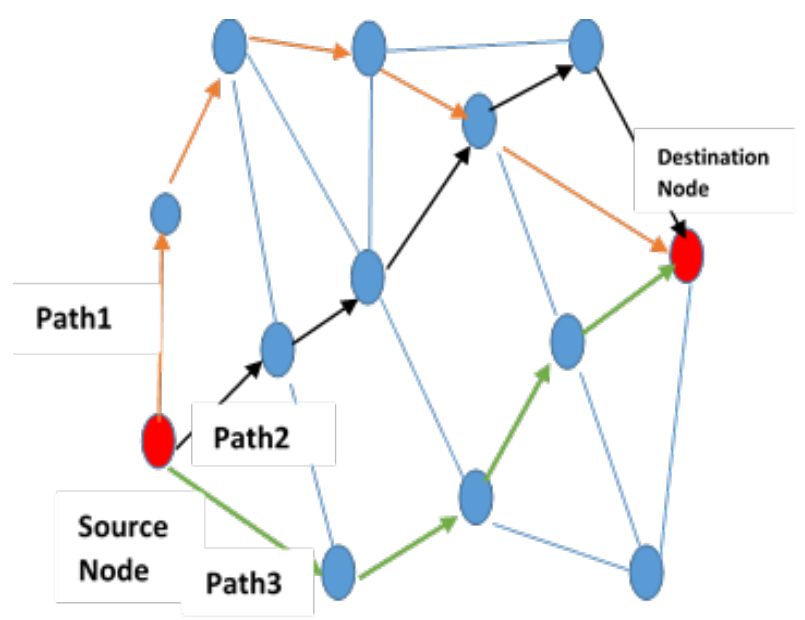

Figu re 3. Designated for multilayer, and protocols

The figure 3 shows that is designated for multilayer, and protocols are applied in mobile ad hoc networks in this protocol is confidential for a different path in different data travel in each root path. Path 1,2,3 in a single source is provided for various data in a single destination node received different data's similar time, MLP is used in these networks reduce duplicate data (refer figure 2), crash and system traffic also minimize use. It is batter for TDMA never for waiting for data broadcast and packets receiving.

\section{A. WMLP}

WMLP is the upper layer protocol material to perform flow classification constraint-based routing or other adapted processing.

Table 1 Variables in algorithm 1

\begin{tabular}{|c|l|}
\hline Symbol & Description \\
\hline IDsn & source node address \\
\hline IDdn & destination node address \\
\hline IDnn & Neighbors of the source node or current node \\
\hline Cs & Source Connection \\
\hline
\end{tabular}




\begin{tabular}{|c|l|}
\hline $\mathbf{C d}$ & Destination Connection \\
\hline $\mathbf{w}$ & web-like networks model \\
\hline $\mathbf{t}$ & The established path tree \\
\hline $\mathbf{T s}$ & Source nodes send time the requesting node \\
\hline $\mathbf{T r}$ & Received node time confirmation \\
\hline
\end{tabular}

\section{Algorithm 1 Path Discovery}

Process path detection (IDsn; IDdn)

If

$$
\mathrm{IDdn}=\mathrm{IDnn} \text { then }
$$

Through packet to IDdn

\section{Else}

Fix the Isn and IDn

Produce the network's web-like model w

Generate the multi-path rout tree $\mathrm{t}$

Efficient rout path search $\mathrm{T}$ and get paths.

Send request nodes

Activate (request Time)

If receive confirmed Network rout path when request Time is not timeout then

Packet delay (Tr-Ts) when receiving established Networks.

Return the way with the minimum (Tr-Ts)

\section{Else}

Restart efficient rout Path Discovery.

\section{End if}

\section{End if}

\section{End process}

\section{RESULT AND DISCUSSIONS}

To study the enactment of the WMLP by using multipath routing protocols. The duplication settings Produced 
the ns2 using some of the specialized tools used to compile and execute programs, tool command language using for ns2 it is in using Ns-allinone-2.28 package it is wireless protocols simulation part of 1200 meters x 1200 meters close distance in check for 10 seconds of simulated time. MAC layer model using for communication done by networks and create a multiple layer rout paths it participated multi packages and its sending to the destination different root path and consider packet size 512 bytes.

Table 2. Parameter Classification

\begin{tabular}{|l|c|}
\hline Parameters & Value \\
\hline Package Name & Ns-allinone 2.28 \\
\hline Algorithm & WMLP \\
\hline Area & $1200 \mathrm{~m} \times 1200 \mathrm{~m}$ \\
\hline Broadcast Area & $250 \mathrm{~m}$ \\
\hline Communication protocols & UDP,CBR \\
\hline Packets size & 512 bytes \\
\hline
\end{tabular}

\section{RATIO GRAPH:}

\section{A. RATIO GRAPH:}

In this method used to improve the overall performance of networks compared from figure 4,5,6,7 whereas efficient throughput, better delivery ratio, strengthen our network delay performance, networking routing enhancement improve and delay to reduce the systems jamming. Here is have used to free buffer model to consume network delay and preclude the interference on a network, compared to existing method better result discovered in this prosed system.

$\mathrm{D}=(\mathrm{Tr}-\mathrm{Ts})$

$\operatorname{Tr}$ - receiving Time

Ts -sending Time 


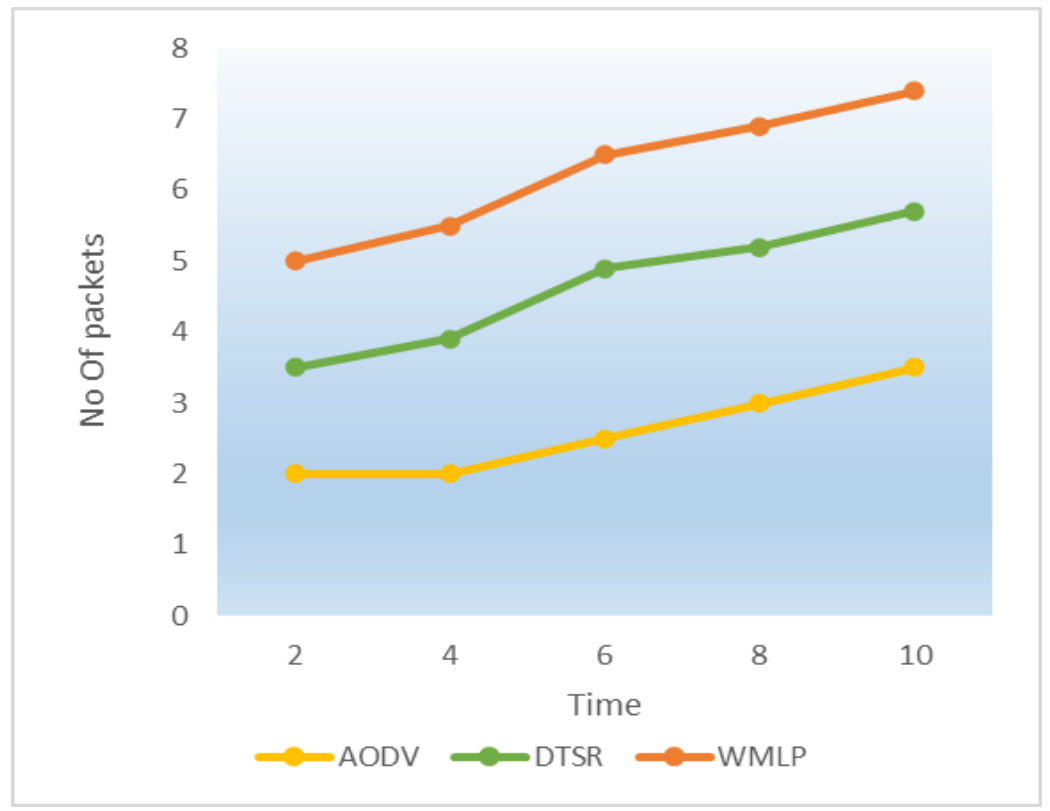

Figure 4.Comparison of throughput

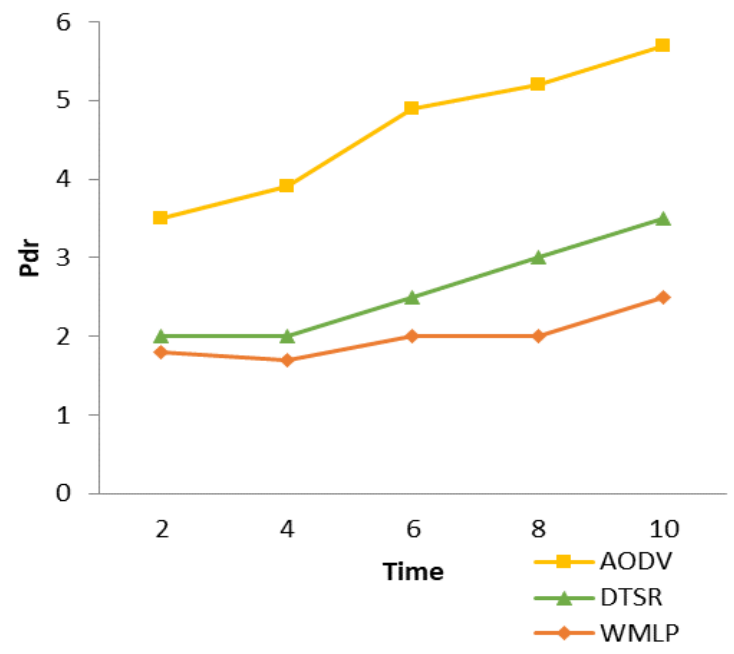

Figu re.5Comparison of packet delay

\section{B. The Data Delivery Fraction}

The data transported from the initial area to purpose on this network. The lively message energy essential for communicating or receiving packets through transmission controller or load distribution, and the energy consumption method added. 


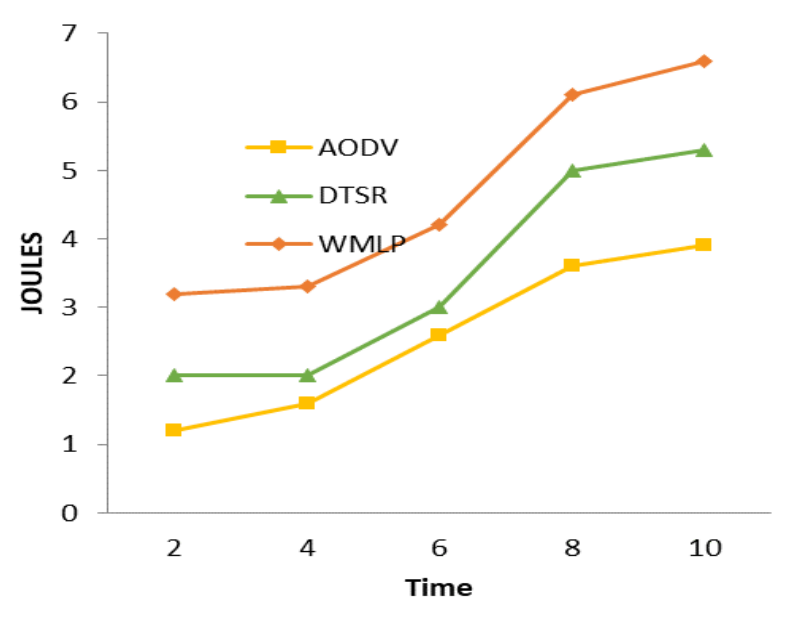

Fig u r e 6.Comparison of the delivery ratio

This is the survey by a two hope between an amount of data received from state hopes to hope the measured package made from initial point onset of connections.

$$
\mathrm{PDF}=(\mathrm{Pr} / \mathrm{Ps})^{*} 100
$$

Where Pr is total no of packets getting \& Ps is the total no of packets have a network or rout path layer.

\section{Energy consumption}

The energy level on the network is the most important one of the fast data transmission on their network. Its calculated from their each node energy consumption is must of the system. If any node none to data transmission that node to save the energy on order. the cluster headtake more heat to send the data from source to destination on the network.

Energy depletion $=$ total no of packets $*$ opening energy level.

Balance energy $=$ energy depletion - Node having total no of packets. 


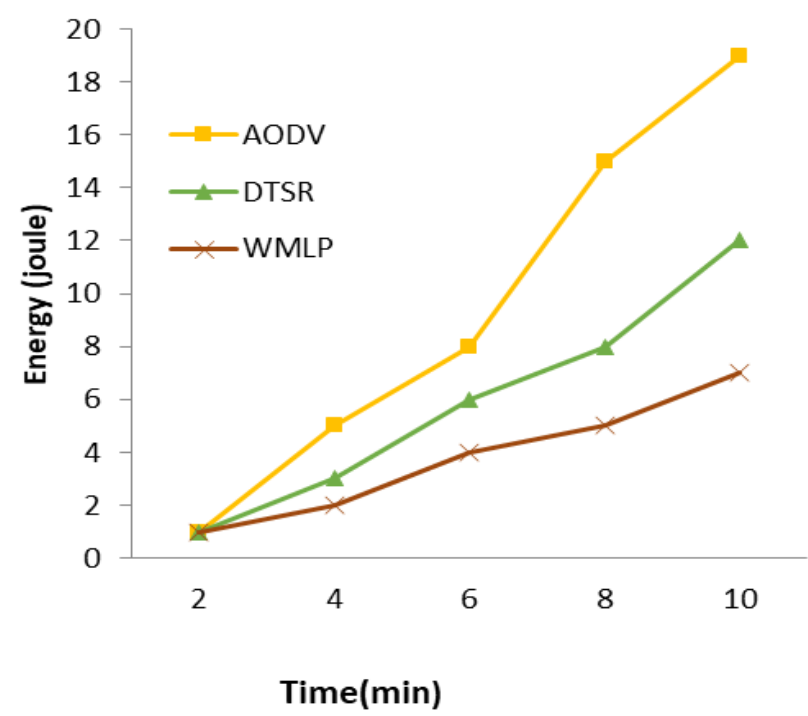

Figure 7. Comparison of Energy consumption on network

\section{CONCLUSIONS}

To satisfy the real-time process of emergency data transmission, in this proposed WMLP done in a wireless network web-based transmission mechanism for emergency data. We create a systems web-based model for searching data restrict the area. Source mobile node sends out request networks, and destination mobile nodes send to response message and an efficient route path in this unauthorized area to launch the connection to well transmission paths. WMLP can reduce the packets delivery delay of emergency data's by using the auto multi-priority queue management technique to function packets. In this way, recover the deadlines for the packages from the consider networks, when transmitted packets we are using restricted containers approach to select the next optimize hop. In this output provide that our proposed WMLP method processed better comparison of another way (ACAR, GPCR, and GPSR) based average overall networks communication delay, packet delivery ratio. The results prove that WMLP can certify real-time necessities of emergency data's level in the occurrence of network traffic.

\section{REFERENCES}

1. O.Gnawali, R. Fonseca, K. Jamieson, D.Moss "Localized Minimum Spanning Tree-Based Multicast Routing with Energy-Efficient Guaranteed Delivery in Ad Hoc and Sensor Networks,",IEEE,978986,2008.

2. Jinglong Zhou, Przemysław Pawełczak, Daniel Wu, "Practical Rate and Route Adaptation with Efficient Link Quality Estimation for IEEE 802.11b/g Multi-Hop Networks",180-186,18 Feb 2010. 
3. Fei Qin Xuewu Dai, John E. Mitchell "Effective-SNR estimation for wireless sensor network using a Kalman filter" ScienceDirect, Volume 11, Issue 3, May 2013, Pages 944-958.

4. Aravind B. Mohanoor, Sridhar Radhakrishnan, Venkatesh Sarangan "On Energy Aware Routing in Wireless Networks",IEEE, pp.609-619, 2004.

5. Qunfeng Dong, Suman Banerjee, Micah Adler "Minimum Energy Reliable Paths Using Unreliable Wireless Links", In ACM. MobiHoc, pages 146-156, June 2002..

6. Ryan C. Kroeze, Philip T. Krein, "Electrical Battery Model for Use in Dynamic Electric Vehicle Simulations",IEEE,1336-1346, 2008.

7. M. Ceriottiet al., "Monitoring heritage buildings with wireless sensor networks: The Torre Aquila deployment," in Proc. IPSN, 2009, pp.277-288.

8. L. Mo et al., "Canopy closure estimates with GreenOrbs: Sustainable sensing in the forest," in Proc. SenSys, IEEE INFOCOM,2009, pp. 99-112.

9. Y. Liu, K. Liu, and M. Li, "Passive Diagnosis for wireless sensor networks," IEEE/ACM Trans. Netw., vol. 18, no. 4, pp. 1132-1144, Aug 2010.

10. O.Gnawali, R. Fonseca, K. Jamieson, D.Moss, and P. Levis, "Collection tree protocol," 7th ACM Conference on Embedded Networked Sensor Systemsin Proc. SenSys, 2009, pp. 1-14.

11. D. S. J. D. Couto, D. Aguayo, J. Bicket, and R. Morris, "A high throughput path metric for multi-hop wireless routing",Proc. MobiCom, pp. 134-146, 2003.

12. Z. Li, M. Li, J. Wang, and Z. Cao, "Ubiquitous data collection for mobile users in wireless sensor networks",Proceedings IEEE INFOCOM, 2246-2254, 2011.

13. X. Lu, D. Dong, Y. Liu, X. Liao, and L. Shanshan, "PathZip: Packet path tracing in wireless sensor networks," in. Proc. IEEE MASS, 2012, pp. 380-388.

14. M. Keller, J. Beutel, and L. Thiele, "routing dynamics in deployed sensor networks with multi-hop network tomography," in Proc. SenSys, ACM,2012, pp. 15-28.

15. Y. Yang, Y. Xu, X. Li, and C. Chen, "A loss inference algorithm for wireless sensor networks to improve data reliability of digital ecosystems," vol. 58, no. 6, pp. 2126-2137, Jun.2011.

16. Saravanan, K. and Ravichandran, T. "An Optimal Rate Adjustment Algorithm for MAC Protocol in 802.11 Multi Hop Ad Hoc Networks” Journal of Computer Science, ISSN 1549-3636 C 2012 Science Publications URL: http://thescipub.com/abstract/10.3844/jcssp. 2012. 1156.1164,Vol.8, No.7, pp. 1156$1164,2012$.

17. Saravanan, K. and Ravichandran, T. "Power Adjustment Algorithm for Higher Throughput in Mobile Ad hoc Networks", International Journal of Computer Science and Network Security. (Journal ISSN 17387906 http://paper.ijcsns.org/07_book/html/201204/201204010. html) Vol.12, No.4, pp.77-85, 2012.

18. Saravanan, K. and Ravichandran, T. "An Efficient Power Utilization Protocol for Achieving Maximum Throughput in Wireless Communication" International Journal of Systems, Algorithms \& Applications. (ISSN Online: 2277-2677 http://www.ijsaa.org) Vol.2, Issue 4, pp. 23-25, April 2012. 
19. Saravanan, K. and Ravichandran, T. "Data Rate Control Algorithm for MAC Protocol in 802.11 Multi Hop Ad Hoc Networks" International Journal of Scientific \& Engineering Research (IJSER) ISSN Online: 2229-5518, http://www.ijser.org, Vol. 3, Issue 5, May 2012.

20. Saravanan, K. S. Ramani and Dr. J. Sundararajan, “A Novel Traffic Aware based Routing Integrated Time Synchronization for Slack Time Minimization in NoC System" Asian Journal of Research in Social Sciences and Humanities Vol. 6, Special Conference Issue Sept 2016, pp. 310-318. ISSN 2249-7315

21. Saravanan, K. S. Elango and Dr. J. Sundararajan, "MNN: Multiclass Neural Network Classifier for Cardiac Disease Prediction Models" Asian Journal of Research in Social Sciences and Humanities, Vol. 6, Special Conference Issue Sept 2016, pp. 293-309. ISSN 2249-7315.

22. Saravanan, K, "Quality of service (QoS) and priority aware models for energy efficient and demand routing procedure in mobile ad hoc networks" Journal of Ambient Intelligence and Humanized Computing- Springer Nature, DOI 10.1007/s12652-020-01769-7, ISSN 1868-5137.

23. Saravanan, K, "A Machine Learning Perspective towards Detecting Fake News" International Journal for Research in Applied Science and Engineering Technology, VOL-8 ISSUE-V, ISSN:2321-9653, DOI:10.22214, May 2020.

24. S.Anthoniraj, S Saraswathi, "Frequent failure monitoring and reporting in virtualization environment using backingalgorithm technique", Int.J. Information and Communication Technology, Vol: 12, No: 1/2, pp: 131-142, 2018.

25. S.Anthoniraj, S Saraswathi, "Hypervisor Monitor Tool in Virtualization Environment Using Hyper Monitor Algorithm Technique", Advances in Natural and Applied Sciences, Vol: 9, No: 6, pp: 331-337, 2015.

26. S.Anthoniraj, S Saraswathi, "Optimized resource and shared allocation using ESUAB algorithm in virtual environment”, Int. J. Advanced Intelligence Paradigms, Vol: 7, No: 3/4, pp: 302-312, 2015.

27. S.Anthoniraj, S Saraswathi, "Resource Sharing For Hypervisor Based Architecture In Virtualization Environment", International Journal of Research in Engineering and Technology, Vol. 3, Special Issue: 7, pp: 107-112, 2014.

28. S.Anthoniraj, S Saraswathi, "Effective Data Security in Virtualization using Maize Routing Technique", Research Journal of Applied Sciences, Engineering and Technology, Vol: 8(6), pp: 755-759, 2014.

29. S.Anthoniraj, S Saraswathi, "Port Progressive Technique in Virtual-Environment using PFT algorithm", International Journal of Applied Engineering Research, Vol: 9, pp: 5028-5032, 2014. 